\title{
Cardiac conditioning in the albino rabbit using 3 CS-UCS intervals
}

\begin{abstract}
Abstraet
The cardiac response was conditioned in rabbits using a trace-conditioning procedure with either 2,4 , or 6 sec. between CS termination and UCS onset. The CR when corrected for the use of a non-neutral CS was a deceleration in rate. The latency of maximum deceleration varied with the length of the CS-UCS interval Problem
\end{abstract}

Although there have been a large number of studies in which successful cardiac conditioning has been reported, the problem regarding the form of the CR seems to be largely unresolved. In humans, Notterman et al (1952) reported finding a deceleration in the interval between the CS and shock while Lacey \& Smith (1954) found an acceleration following verbal stimuli which had been paired with shock. Moreover, Zeaman et al (1954) reported finding an acceleration followed by a deceleration during a 6-sec. CS-UCS interval.

The findings from animal studies are also variable with some investigators such as Dykman \& Gantt (1959) finding an acceleration in dogs while others such as Black et al (1962) reporting different responses in different dogs with a further suggestion that a delayed conditioning procedure is more likely to lead to acceleration and a trace conditioning procedure to an acceleration followed by deceleration in the CS-UCS interval.

Unfortunately, comparisons among the results of these cardiac conditioning studies are made difficult due to the wide differences in procedure and in species employed. The present study is one of a series designed to explore systematically cardiac conditioning in the albino rabbit.

\section{Method}

Eighteen 150-180 day old experimentally naive albino rabbits who had been handled daily for 2 months were adapted to a restraining box for 2 weeks before experimentation. Two days before conditioning trials began three stainless steel safety pin electrodes were inserted for EKG recording and each $S$ was then placed for $1 \mathrm{hr}$. on two successive days in the ventillated sound-treated chamber where the conditioning trials were to be given.

The actual five-day conditioning sequence was as follows: On Day 1 a 1-hr. period of adaptation in the chamber was followed by 20 preconditioning trials of CS alone and then by $20 \mathrm{CS}-\mathrm{UCS}$ pairings. On Days 2-5 the 1-hr. adaptation period was followed by 20 CS-UCS pairings. Thus, all Ss were given a total of 100 conditioning trials. For all Ss, the CS was a 1-sec. $1000 \mathrm{cps}$ tone at $80 \mathrm{db}$ delivered through a

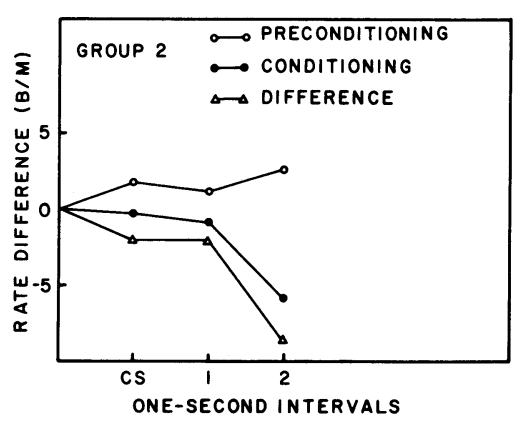

Fig. 1. Mean cardiac response to the $\mathrm{CS}$ during preconditioning trials 10-20, conditioning trials 1-100, and the corrected CR (difference curve) for Group 2.

speaker 12 in in front of the S's head. The UCS was a 1-sec. 3 ma constant current shock applied to the grid floor of the restraining box through a scrambler. For Groups 2, 4, and 6 the interval between CS termination and UCS onset was 2, 4, and 6 sec. respectively. Each group consisted of three male and three female Ss.

During the preconditioning and conditioning trials, which were spaced at a mean intertrial interval of 2 min., recordings were taken from $10 \mathrm{sec}$. before CS onset to $15 \mathrm{sec}$. after CS termination. All timing was done automatically and recordings were made on a Grass model 5 polygraph using 5P4 and 5P1 preamplifiers and a chart speed of $100 \mathrm{~mm} / \mathrm{sec}$.

\section{Results}

For each of the preconditioning and conditioning trials the pre-tone heart rate was determined over the 6-sec. period immediately preceding CS onset. During the CS and during each of the following 1-sec. intervals of time, the first complete $R$ to $R$ interval was measured and converted to rate in $\mathrm{b} / \mathrm{m}$. The pre-tone rate was then subtracted from each of these values in order to show the rate change brought about by CS presentation. In Fig. 1 the curve with the open circles shows for Group 2 the mean cardiac responses to the CS taken over the last 10 preconditioning trials. The curve with the filled circles shows the mean cardiac response to the CS taken over all 100 conditioning trials. Since there was a cardiac change to the CS alone during the preconditioning trials, the CR was determined by subtracting the preconditioning $R$ to the $C S$ from the response to the CS obtained on the conditioning trials. The curve with the open triangles in Fig. 1 shows the result of this correction. For this group the correction results in an increase in the amplitude of the deceleration. 


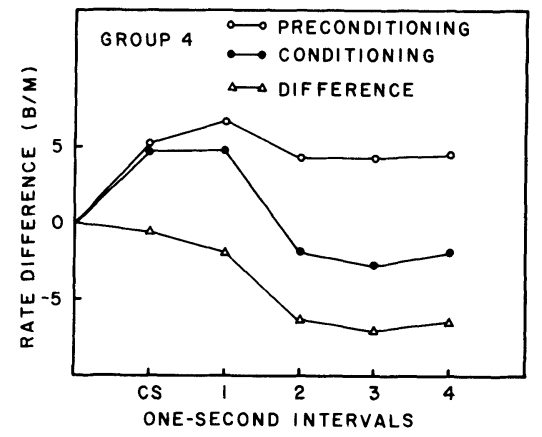

Fig. 2. Mean cardiac response to the $\mathrm{CS}$ during preconditioning trials 10-20, conditioning trials 1-100, and the corrected CR (difference curve) for Group 4

Figure 2 shows the results of this same calculation for Group 4. Note that the apparent acceleration component of the response disappears when the correction for the non-neutral CS is applied.

In Fig. 3 the results for Group 6 are presented in the same manner and again the corrected response is a deceleration in rate.

The use of the form of the response to the CS on preconditioning trials 11 to 20 as a correction factor in determining the form of the CR requires the assumption that the form of the response to the CS would remain constant over the following 100 trials if shock were not introduced. The results of a study in progress in which Ss are given 120 tone-alone trials in the same temporal sequence as in the present study seems to substantiate this assumption.

In order to compare the forms of the CR, the corrected CR for all groups is shown in Fig. 4 Notice that as the CS-UCS interval is lengthened from 2 to 6 sec., the latency of the maximum deceleration as indicated by the arrows also increases. This difference in latency of maximum deceleration is significant at better than the .01 level $(\mathrm{H}=11.95 ; \mathrm{df}=2)$ using the Kruskal-Wallis test. The mean deceleration over all 100 trials at each of these three maximum points is indicated beside the arrows and is $8.4 \mathrm{~b} / \mathrm{m}$ for Group $2,7.1 \mathrm{~b} / \mathrm{m}$ for Group 4 , and $5.5 \mathrm{~b} / \mathrm{m}$ for Group 6. An $\mathbf{F}$ test done on these maximum deceleration values over blocks of 20 conditioning trials does not result in a significant CS-UCS interval effect, however. Only the Trials effect is

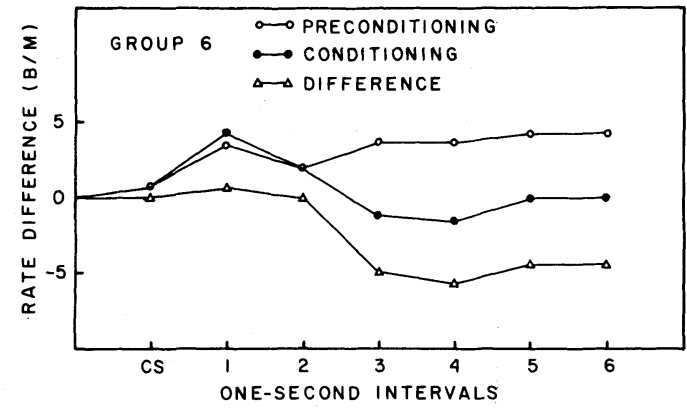

Fig. 3. Mean cardiac response to the $\mathrm{CS}$ during preconditioning trials 10-20, conditioning trials 1-100, and the corrected CR (difference curve) for Group 6.

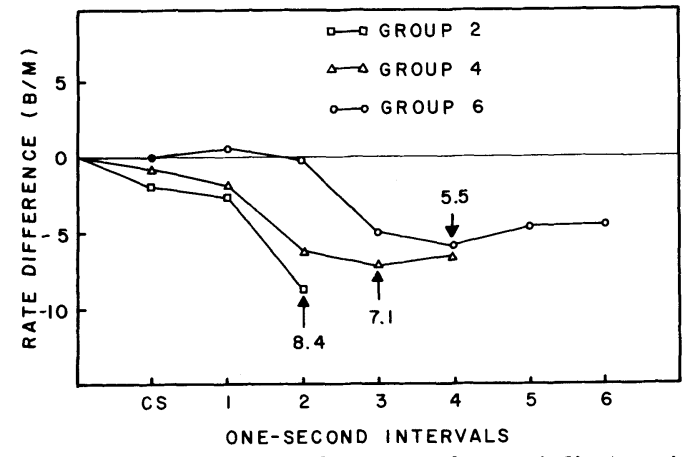

Fig. 4. Corrected CR for all groups. Arrows indicate points of maximum deceleration over all 100 conditioning trials.

significant at better than the .05 level $(F=2.75 ; \mathrm{df}=4,60)$. For all groups the maximum deceleration occurs during the 4th block of conditioning trials with a slight drop in amplitude during the 5 th block of 20 trials.

\section{Diseussion}

The present study indicates that the cardiac response can be conditioned in the rabbitusing a trace-conditioning procedure, and that this response is a deceleration in rate. Although the amplitude of the deceleration did not differ significantly among groups, the latency of the point of maximum deceleration increased with the length of the CS-UCS interval, thus showing Pavlovian inhibition of delay.

It should be noted that the response to the CS had not adapted out prior to the beginning of conditioning and that if a correction had not been made for the use of this non-neutral CS, the form of the CR in Groups 4 and 6 would have been the diphasic one of acceleration followed by deceleration. It seems probable that some of the difficulties regarding specification of form of the cardiac $\mathrm{CR}$ have resulted from differences in the assumptions made regarding the nature of the original response to the CS and its change with repeated applications. Tones, which have been employed frequently as the $\mathrm{CS}$ in cardiac conditioning studies, have been shown to have various effects upon cardiac rate depending upon their intensity, duration, and frequency of application. If continued use of tones is to be made in these studies, additional parametric studies of the unconditioned cardiac response to them would be valuable.

\section{References}

Black, A. H., Carlson, N. J., \& Solomon, R. L. Exploratory studies of the conditioning of autonomic responses in curarized dogs. Psychol. Monogr., 1962, 76, No. 29 (Whole No. 548).

Dykman, R. A., \& Gantt, W. H. The parasympathetic component of unlearned and acquired cardiac responses. J. comp. physiol. Psychol., 1959, 52, 163-167.

Lacey, J. I., \& Smith, R. L. Conditioning and generalization of unconscious anxiety. Science, 1954, 120, 1045-1052.

Notterman, J. M., Schoenfeld, W. N., \& Bersh, P. J. Conditioned heart rate response in human beings during experimental anxiety. J. comp. physiol. Psychol., 1952, 45, 1-8.

Zeaman, D., Deane, G. E., \& Wegner, N. Amplitude and latency characteristics of the conditioned heart response. J. Psychol., $1954,38,235-250$.

\section{Note}

1. This research was supported in part by Grant MH-06590-03 from the National Institutes of Health. 